\title{
¿Son efectivos los repelentes contra mosquitos para prevenir enfermedades transmitidas por vectores?
}

\section{Are mosquito repellant agents effective for preventing vector-borne diseases?}

Correspondencia

Nelly Godoy

nelly.godoy.mendoza@gmail.com

Recibido: 24/10/2016

Aprobado: 09/11/2016

Citar como: Godoy N, Gutierrez EL, Hijar G. ¿Son efectivos los repelentes contra mosquitos para prevenir enfermedades transmitidas por vectores? Acta Med Peru. 2016;33(4):346-7

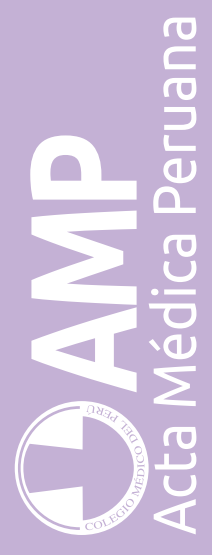

\author{
Nelly Godoy ${ }^{1 a}$, Ericson L. Gutierrez ${ }^{1,2}$, Gisely Hijar ${ }^{1 b}$ \\ 1 Unidad de Análisis y Generación de Evidencias en Salud Pública, Instituto Nacional de Salud. Lima, Perú. \\ 2 Facultad de Medicina, Universidad de San Martin de Porres. Lima, Perú. \\ a Licenciada en enfermería; b Bióloga
}

Sr. Editor:

En la actualidad, las enfermedades transmitidas por vectores como el dengue, zika y Chikungunya representan problemas de salud pública. La utilización de repelentes se considera una medida de prevención contra las picaduras de mosquitos transmisores de éstas enfermedades ${ }^{[1]}$.

En el mercado internacional circulan dos clases de repelentes, los sintéticos como el DEET (N,N-Diethyl-meta-toluamide), la icaridina (Picaridín, Bayrepel o KBR3023) y el IR3535 (EBAAP: ethyl butylacetylaminopropionate); y los naturales como el aceite de eucalipto limón (Corymbia citriodora) y el aceite de citronela. Sin embargo, sólo el DEET, el IR3535 y la icaridina han sido aprobados por la Agencia de Protección Ambiental de los Estados Unidos (EPA, por sus siglas en inglés) y al mismo tiempo son recomendados por la Organización Mundial de la Salud (OMS) como repelentes efectivos ${ }^{[2]}$.

Una revisión analizó 102 publicaciones entre los años 2000 y el 2012 que evaluaron la eficacia del DEET (20 - 25\%) comparado con la icaridina (10 - 20\%) e IR3535 (10 - 20\%), mostrando que el DEET al $20 \%$ ofrecía una protección completa hasta por 10 horas frente al Aedes aegypti, el IR3535 al 20\%, 10 horas y en menor proporción la icaridina al $19,2 \%$, (hasta 8 horas) ${ }^{[3]}$. Sin embargo, ensayos de campo hechos en Australia usando icaridina al 19,2\% y DEET al $35 \%$ dos veces al día, señalan a la icaridina como mejor aceptada ya que sería menos grasosa y pegajosa que el DEET ${ }^{[4]}$.

No se han reportado efectos tóxicos notorios para repelentes como DEET, icaridina o IR3535 a concentraciones recomendadas, por lo que la OMS no restringe su uso en gestantes y niños ${ }^{[2]}$. El DEET ha sido el repelente más estudiado en cuanto a reacciones adversas, pero la mayoría de las reacciones que fueron reportadas estuvieron asociadas a ingestión del químico o por el uso de concentraciones altas sobre la piel (70\%), por ello desde su aprobación sigue siendo el repelente de elección y es considerado como el Gold standard ${ }^{[5]}$.

Los nombres comerciales, el tiempo de protección y las concentraciones más comunes de los repelentes disponibles en nuestro medio se muestran en la Tabla $1^{[1]}$.

Respecto al uso simultáneo de protector solar (como oxibenzona) y repelente (DEET o icaridina), este no es recomendable, ya que tienen distintos mecanismos de acción y frecuencia de uso; pudiendo incrementar la absorción de ambos químicos o reduciendo la penetración dérmica; y con ello un riesgo de reacciones en la piel, por lo que se sugiere primero aplicar el protector solar y después de unos diez a veinte minutos aplicar el repelente, y reaplicar según indique la etiqueta del producto ${ }^{[6]}$. 
Tabla 1. Tiempo de duración del efecto protector de los repelentes ${ }^{[1]}$.

\begin{tabular}{|c|c|c|c|}
\hline Ingrediente principal & Nombre comercial & Tiempo de duración & $\begin{array}{l}\text { Formulación/ } \\
\text { presentación }\end{array}$ \\
\hline DEET $<10 \%$ & Skintastic (Off), Cutter, Skedaddle. & $1-3$ horas & $\begin{array}{l}\text { Loción, espray, gel, } \\
\text { aerosol. }\end{array}$ \\
\hline DEET $10-30 \%$ & $\begin{array}{c}\text { Cutter, Cutter Backwoods, Cutter } \\
\text { Backyard, Deep Woods Off! Muskol, Off! } \\
\text { utdoorsman. }\end{array}$ & $4-6$ horas & $\begin{array}{l}\text { Loción, espray, } \\
\text { aerosol. }\end{array}$ \\
\hline DEET $20-33 \%$ & Sawyer, Ultrathon & $6-12$ horas & Loción, aerosol \\
\hline Icaridin $7 \%$ & Cutter advanced & $3-4$ horas & Espray. \\
\hline Icaridin $15 \%$ & Cutter advanced sport & $6-8$ horas & Aerosol \\
\hline IR35335 & Antimosquito (Johnson \& Johnson) & $4-6$ horas & Loción \\
\hline
\end{tabular}

En el Perú es posible encontrar productos comerciales que contienen DEET, icaridina o IR3535. La capacidad de repelencia del compuesto depende de la concentración y el tiempo de exposición.

En conclusión, DEET, icaridina e IR3535 son tres repelentes efectivos en brindar protección contra la picadura de mosquitos como el Aedes aegypti. Los estudios han demostrado que son seguros de aplicar en la población general, incluyendo gestantes a partir del segundo trimestre de gestación y niños mayores de 2 meses de edad, pero tomando en cuenta las indicaciones del fabricante de cada producto. La aplicación de éstos repelentes podría ser una estrategia efectiva para prevenir la transmisión de enfermedades como el dengue, chikungunya y zika en la población.

Fuente de financiamiento: El presente artículo ha sido financiado por el Instituto Nacional de Salud.

Conflicto de intereses: Los autores declaran no tener conflictos de interés con respecto a la publicación del presente artículo.

\section{REFERENCIAS BIBLIOGRÁFICAS:}

1. Commitee to Advise on Tropical Medicine and Travel (CATMAT). Statement on personal protective measures to prevent arthropod bites. Can Commun Dis Rep. 2005;31(ACS-4):1-18.

2. Katz TM, Miller JH, Hebert AA. Insect repellents: historical perspectives and new developments. J Am Acad Dermatol. 2008;58(5):865-71.

3. Lupi $E_{1}, \mathrm{Hatz} C$, Schlagenhauf P. The efficacy of repellents against Aedes, Anopheles, Culex and Ixodes spp. - a literature review. Travel Med Infect Dis. 2013;11(6):374-411.

4. Scheinfeld N. Picaridin: a new insect repellent. Med Lett Drugs Ther. 2005;47(1210):46-7.

5. Koren G, Matsui D, Bailey B. DEET-based insect repellents: safety implications for children and pregnant and lactating women. CMAJ. 200;169(3):209-12.

6. Rodriguez J, Maibach HI. Percutaneous penetration and pharmacodynamics: Wash-in and wash-off of sunscreen and insect repellent. J Dermatolog Treat. 2016;27(1):11-8.

\section{Ahora puede enviar sus artículos para Acta Médica Peruana en nuestro Open Journal System:}

\section{www.amp.cmp.org.pe}

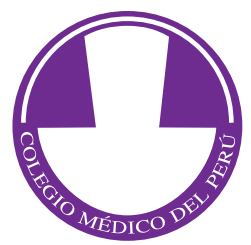

